ARTICLE

Received 7 Nov 2016 | Accepted 28 Mar 2017 | Published 19 May 2017

DOI: $10.1038 /$ ncomms15417

OPEN

\title{
Energy landscape-driven non-equilibrium evolution of inherent structure in disordered material
}

Yue Fan', Takuya Iwashita² \& Takeshi Egami $3,4,5,6$

Complex states in glasses can be neatly expressed by the potential energy landscape (PEL). However, because PEL is highly multi-dimensional it is difficult to describe how the system moves around in PEL. Here we demonstrate that it is possible to predict the evolution of macroscopic state in a metallic glass, such as ageing and rejuvenation, through a set of simple equations describing excitations in the PEL. The key to this simplification is the realization that the step of activation from the initial state to the saddle point in PEL and the following step of relaxation to the final state are essentially decoupled. The model shows that the interplay between activation and relaxation in PEL is the key driving force that simultaneously explains both the equilibrium of supercooled liquid and the thermal hysteresis observed in experiments. It further predicts anomalous peaks in truncated thermal scanning, validated by independent molecular dynamics simulation.

\footnotetext{
${ }^{1}$ Department of Mechanical Engineering, University of Michigan, Ann Arbor, Michigan 48109, USA. ${ }^{2}$ Division of Natural Sciences, Oita University, Oita-shi 870-1192, Japan. ${ }^{3}$ Shull Wollan Center - Joint Institute for Neutron Sciences, Knoxville, Tennessee 37831, USA. ${ }^{4}$ Department of Materials Science and Engineering, University of Tennessee, Knoxville, Tennessee 37996, USA. ${ }^{5}$ Department of Physics and Astronomy, University of Tennessee, Knoxville, Tennessee 37996, USA. ${ }^{6}$ Materials Science and Technology Division, Oak Ridge National Laboratory, Oak Ridge, Tennessee 37831, USA. Correspondence and requests for materials should be addressed to Y.F. (email: fanyue@umich.edu).
} 
G lasses do not have structural defects found in crystalline materials, such as dislocations and grain boundaries. For this reason glasses have many promising physical, chemical and mechanical properties in comparison to their crystalline counterparts $^{1-3}$, making them attractive for a wide range of applications. Many properties of glassy materials are very sensitive to the degree of relaxation in the systems, which can be expressed in terms of their inherent structure (IS) obtained by removing the kinetic energy ${ }^{4-6}$. However, a fundamental understanding is still lacking on the dynamics of IS and its connection with the properties of glasses such as ageing (e.g., decrease in the IS energy) or rejuvenation (e.g., increase in the IS energy). The challenge is twofold: firstly, glasses represent complex non-equilibrium states of matter that contain manybody interactions and strong disorder. This makes it difficult to develop parameter-free theories based on defects, because the definitions of defects in disordered materials are not unique and would require some phenomenological presumptions and fitting parameters $^{7-9}$. Secondly, although computational modeling have yielded notable advances in our knowledge of complex materials at the atomistic scale, the traditional techniques (e.g., molecular dynamics (MD)) can only tackle problems at limited timescales and therefore face formidable challenges in probing materials behavior far from equilibrium.

The IS and the potential energy landscape (PEL) are known to be capable of providing a convenient framework to interpret complex phenomenology in disordered materials ${ }^{10-13}$, and more importantly, they allow focusing directly on energy variations, making it easier to compare with experiments (e.g., differential scanning calorimetry (DSC)) with minimal fitting parameters. In the PEL perspective, the elementary processes are the hopping between neighboring local minima. This consists of two stages; the activation stage (from the initial state to the connecting saddle state with the activation barrier $E_{\mathrm{A}}$ ), and the relaxation stage (from the saddle state to the final state with the energy relaxation $E_{\mathrm{R}}$ ), as illustrated in Fig. 1. Assuming at time $t$ the IS energy of the system is $E_{\mathrm{IS}}(t)$, one can then write down its evolution equation as:

$$
\begin{aligned}
\frac{\mathrm{d} E_{\mathrm{IS}}}{\mathrm{d} t} & =\frac{E_{\mathrm{IS}}(t+\Delta t)-E_{\mathrm{IS}}(t)}{\Delta t} \\
& =v \cdot \exp \left(-\frac{E_{\mathrm{A}}}{k_{\mathrm{B}} T}\right) \cdot\left[E_{\mathrm{A}}-E_{\mathrm{R}}\right]
\end{aligned}
$$

where $\Delta t=\left[v \cdot \exp \left(-\frac{E_{\mathrm{A}}}{k_{\mathrm{B}} T}\right)\right]^{-1}$ represents the waiting time for hopping, and $v$ is the jump frequency which includes the entropic effect. Note that Fig. 1 is only a schematic illustration, and in disordered materials, each IS is connected to many different saddle states. Therefore all the possibilities need to be considered and equation (1) has to be solved in a mean-field sense as:

$$
\frac{\mathrm{d} E_{\mathrm{IS}}}{\mathrm{d} t}=v \cdot \exp \left(-\frac{\bar{E}_{\mathrm{A}}}{k_{\mathrm{B}} T}\right) \cdot\left[\bar{E}_{\mathrm{A}}-\bar{E}_{\mathrm{R}}\right]
$$

where the details of $\bar{E}_{\mathrm{A}}$ and $\bar{E}_{\mathrm{R}}$ are discussed below. Given equation (2) one can probe how the IS of a glassy system evolves with time under different thermal protocols. In particular, whether the system ages or rejuvenates is determined by the sign of $\left(\bar{E}_{\mathrm{A}}-\bar{E}_{\mathrm{R}}\right)$ : a negative sign corresponds to ageing (IS energy loss), while a positive sign represents rejuvenation (IS energy gain). In the following sections, we will identify the corresponding conditions where the system would undergo ageing, exhibit rejuvenation, or keep itself in the equilibrium state.

\section{Results}

Spectra of $\boldsymbol{E}_{\mathrm{A}}$ and $\boldsymbol{E}_{\mathbf{R}}$. To solve equation (2) we first have to know the dependence of the activation barrier $\left(E_{\mathrm{A}}\right)$ and the energy of

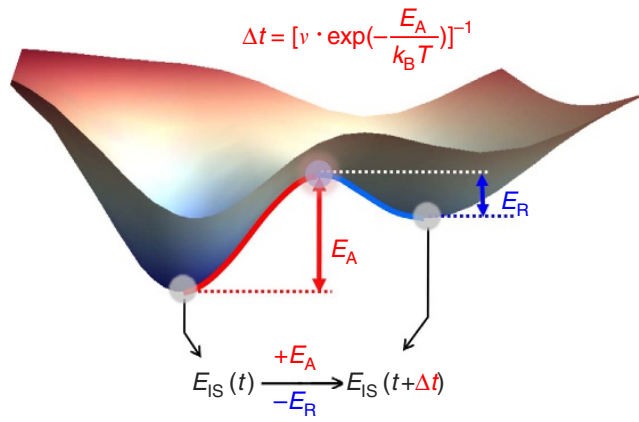

Figure 1 | Schematic illustration of elementary hopping in potential energy landscape (PEL). The evolution of inherent structure (IS) energy is determined by the interplays between activation stage and relaxation stage in each elementary hopping. The $E_{1 S}$ change after hopping is given by $E_{\mathrm{A}}-E_{\mathrm{R}}$. Note that in disordered materials, each IS is connected to many different saddle states. Therefore in real calculation all the possibilities need to be considered, as discussed below in the text.

relaxation $\left(E_{\mathrm{R}}\right)$ on $E_{\mathrm{IS}}$. Due to its disordered nature in glasses, both $E_{\mathrm{A}}$ and $E_{\mathrm{R}}$ are expected to display wide spectra, rather than few explicit values as in their crystalline counterparts. These spectra are obtained by the activation-relaxation technique $(\mathrm{ART})^{14,15}$ - a method of atomistic simulation known to be capable of capturing saddle point states and providing representative PEL samplings in amorphous systems, including high activation barrier events which are not accessible by MD simulations ${ }^{16,17}$. As an example here we consider a well-known glass former system $\mathrm{Cu}_{56} \mathrm{Zr}_{44}$ containing 2,000 atoms with a realistic many-body interacting potential ${ }^{18}$. The system is first equilibrated in a high temperature $(2,000 \mathrm{~K})$ liquid state and then cooled to $0 \mathrm{~K}$ with six different thermal conditions, including instant quench (IQ) and cooling with various rates ranging from $10^{13} \mathrm{~K} \mathrm{~s}^{-1}$ down to $10^{9} \mathrm{~K} \mathrm{~s}^{-1}$. Under such protocols six samples with the same composition but very different $E_{\mathrm{IS}}$ are obtained: slower cooling rates lead to lower IS energies. The samples are then input into ART to probe the $E_{\mathrm{A}}$ and $E_{\mathrm{R}}$ spectra.

Figure 2a shows the activation energy spectra obtained for these samples. Not surprisingly faster cooling rates yield smaller average $E_{\mathrm{A}}$, and similar behavior has also been observed in other different glassy systems ${ }^{16,17,19-25}$. More interestingly, it appears that the spectra can be decomposed into two different modes: an exponentially decaying mode at low energies (M1), and a shiftedRayleigh distribution with a clear peak at higher energies (M2). Moreover, the decay rate of M1 and the width of M2 are nearly independent of the cooling rate. Given this the probability distribution function (PDF) of $E_{\mathrm{A}}$ can then be expressed as:

$$
\begin{aligned}
P\left(E_{\mathrm{A}} \mid E_{\mathrm{IS}}\right)= & P_{\mathrm{M} 1}\left(E_{\mathrm{A}} \mid E_{\mathrm{IS}}\right)+P_{\mathrm{M} 2}\left(E_{\mathrm{A}} \mid E_{\mathrm{IS}}\right) \\
= & W\left(E_{\mathrm{IS}}\right) \cdot \frac{1}{\varepsilon_{\mathrm{A}}} \exp \left[-\frac{E_{\mathrm{A}}}{\varepsilon_{\mathrm{A}}}\right] \\
& +W^{\prime} \cdot E_{\mathrm{A}} \cdot \exp \left[-\frac{\left(E_{\mathrm{A}}-\mu\left(E_{\mathrm{IS}}\right)\right)^{2}}{2 \sigma^{2}}\right],
\end{aligned}
$$

where $\varepsilon_{\mathrm{A}}=0.5 \mathrm{eV}, \sigma=0.49 \mathrm{eV}, W\left(E_{\mathrm{IS}}\right)$ is the amplitude of M1, and $\mu\left(E_{\mathrm{IS}}\right)$ is the location parameter of M2. Note that $W\left(E_{\mathrm{IS}}\right)$ and $\mu\left(E_{\mathrm{IS}}\right)$ are the only two independent variables to define the PDF explicitly in equation (3), because $W^{\prime}$ (the relative weight of M2) can be calculated according to normalization condition of the PDF.

As seen in Fig. 2b, when the IS energy becomes lower (i.e., at a slower cooling rate), the amplitude of M1 becomes smaller and the peak of M2 shifts gradually to larger values. An interesting point we would like to note is that, although $W\left(E_{\mathrm{IS}}\right)$ decreases 
a
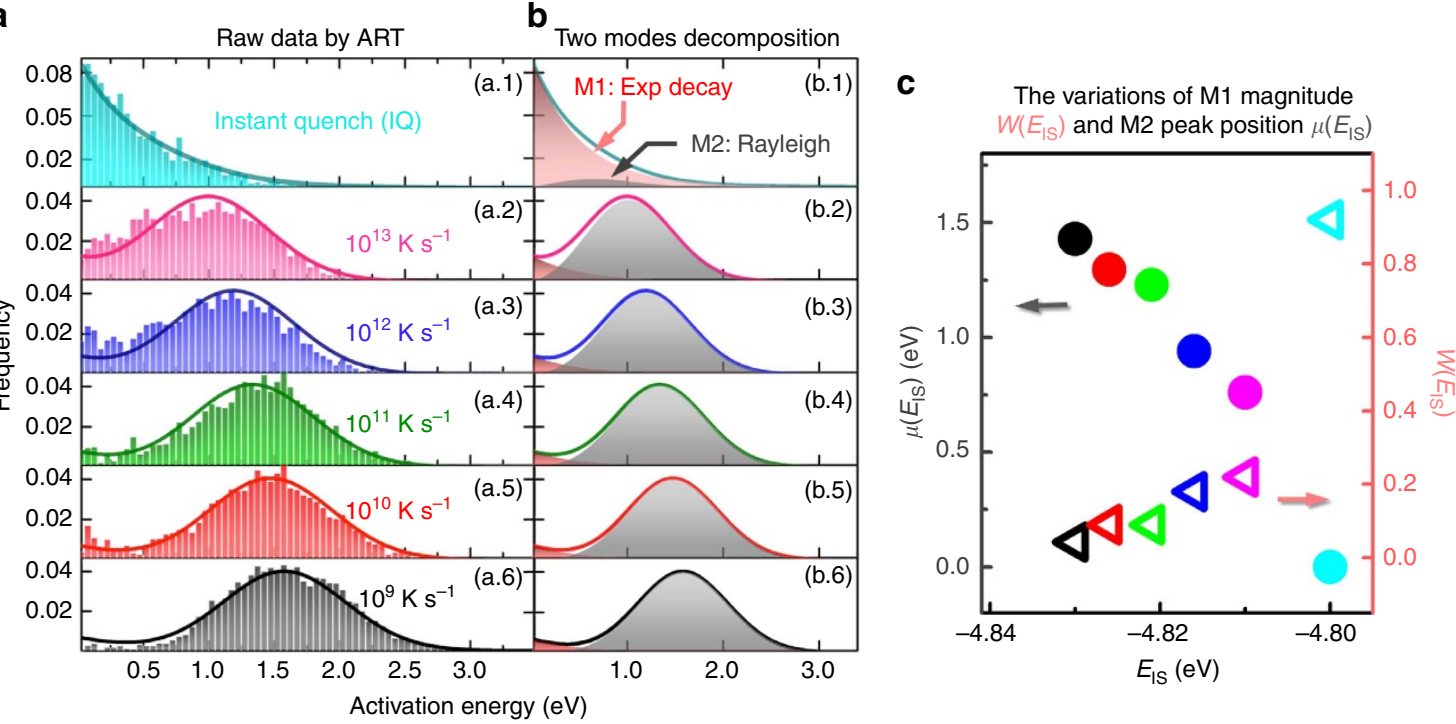

Figure 2 | Dependence of activation energy spectra on cooling rates and EIS. (a) The raw histogram data on the activation barrier spectra in different samples obtained by ART. (b) The spectra can be decomposed into two modes, namely an exponential decay mode (M1), and a shifted-Rayleigh distribution (M2), whose expressions are displayed in c. The magnitude of M1, defined as $W$, and peak position of $M 2$, defined as $\mu$, are closely related to the system's IS energy. As IS energy decreases, $W\left(E_{1 S}\right)$ becomes smaller, while $\mu\left(E_{1 S}\right)$ becomes larger.

very rapidly in the high cooling rate regime, it never disappears at low cooling rates. In the present study, M1 still persists with a fraction around $5 \%$ in the total weight even at the cooling rate of $10^{9} \mathrm{~K} \mathrm{~s}^{-1}$. Such persistence of M1 is probably a characteristic of $\mathrm{ZrCu}$-based glassy system, since experiments ${ }^{26}$ also found extra low activation energy distributions in the same system. However in other systems, e.g., $\mathrm{Pd}_{80} \mathrm{Si}_{20}$ (ref. 26) and amorphous $\mathrm{Si}$ (ref. 17), there are no indications of the M1 mode.

In summary if the values of $W\left(E_{\mathrm{IS}}\right)$ and $\mu\left(E_{\mathrm{IS}}\right)$ are provided, one can then determine the PDF in equation (3), which would further enable the calculation of the average barrier height in the thermally activated processes as:

$$
\bar{E}_{\mathrm{A}}\left(E_{\mathrm{IS}}, T\right)=-k_{\mathrm{B}} T \cdot \ln \left[\int P\left(E_{\mathrm{A}} \mid E_{\mathrm{IS}}\right) \mathrm{e}^{-\frac{E_{\mathrm{A}}}{k_{\mathrm{B}} T}} \mathrm{~d} E_{\mathrm{A}}\right] .
$$

Due to the Boltzmann factor, the average activation barrier now becomes a function of both the IS energy and temperature. Note that equation (4) essentially represents the average of statistically independent events. Although it is an approximation, its validity and rationalization have been extensively discussed by Derlet et al. ${ }^{27,28}$.

As mentioned above, to solve equation(2) the spectrum of energy relaxation $E_{\mathrm{R}}$ also needs to be known. Interestingly, in contrast to $E_{\mathrm{A}}$, the spectrum of $E_{\mathrm{R}}$ was found to be quite insensitive to the IS energy (Supplementary Fig. 1), as was found in the simulation for amorphous Si (ref. 17). The significance of this point will be discussed below. We found that the spectra in all six samples show essentially the same exponential decay distribution,

$$
P\left(E_{\mathrm{R}}\right)=\frac{1}{\varepsilon_{\mathrm{R}}} \exp \left[-\frac{E_{\mathrm{R}}}{\varepsilon_{\mathrm{R}}}\right],
$$

With $\varepsilon_{\mathrm{R}}=0.32(\mathrm{eV})$. Therefore the average energy of relaxation can be calculated as:

$$
\bar{E}_{\mathrm{R}}=\int E_{\mathrm{R}} P\left(E_{\mathrm{R}}\right) \mathrm{d} E_{\mathrm{R}}=\varepsilon_{\mathrm{R}}=0.32 \mathrm{eV} .
$$

Note that there is no Boltzmann factor in equation (6), because the transition from the saddle state to the final state is only downhill athermal relaxation ${ }^{29}$, not a thermally activated process.
Ageing-rejuvenation crossover. In equation (2) whether the system undergoes ageing $\left(\mathrm{d} E_{\mathrm{IS}} / \mathrm{d} t<0\right)$ or rejuvenation $\left(\mathrm{d} E_{\mathrm{IS}} / \mathrm{d} t>0\right)$ depends on the sign of the difference between $\bar{E}_{\mathrm{A}}$ and $\bar{E}_{\mathrm{R}}$ (defined as $\Delta \equiv \bar{E}_{\mathrm{A}}-\bar{E}_{\mathrm{R}}$ ). The ageing-rejuvenation crossover boundary is then governed by the balancing of activation and relaxation shown below in equation (7):

$$
\begin{aligned}
0 & =\Delta\left(E_{\mathrm{IS}}, T\right)=\bar{E}_{\mathrm{A}}-\bar{E}_{\mathrm{R}}=\bar{E}_{\mathrm{A}}\left(E_{\mathrm{IS}}, T\right)-\bar{E}_{\mathrm{R}} \\
& =-k_{\mathrm{B}} T \cdot \ln \left[\int P\left(E_{\mathrm{A}} \mid E_{\mathrm{IS}}\right) \mathrm{e}^{-\frac{E_{\mathrm{A}}}{k_{\mathrm{B}} T}} \mathrm{~d} E_{\mathrm{A}}\right]-\varepsilon_{\mathrm{R}} .
\end{aligned}
$$

In other words, the solutions to equation (7) define a curve in $E_{\mathrm{IS}}-T$ space, on which the system is in equilibrium. As discussed above, if the forms of $W\left(E_{\mathrm{IS}}\right)$ and $\mu\left(E_{\mathrm{IS}}\right)$ are provided, one can readily solve equation $(7)$ and thus predict the equilibrium line. In the present study we have six different samples, the analyses of which show very clear and consistent trend, namely the decrease in $W\left(E_{\mathrm{IS}}\right)$ and the increase in $\mu\left(E_{\mathrm{IS}}\right)$ as $E_{\mathrm{IS}}$ becomes smaller at lower cooling rates (seen in Fig. 2c). However we only have six data points, with inevitable statistical errors embedded in ART calculations, which are insufficient in determining the accurate forms of $W\left(E_{\mathrm{IS}}\right)$ and $\mu\left(E_{\mathrm{IS}}\right)$. Ideally we should be able to gain more knowledge by preparing many more samples in a wider range of $E_{\mathrm{IS}}$, particularly those quenched at slower rates, and by repeating the analyses similarly done in Fig. 2. In principle, as stated by Kallel et al. ${ }^{17}$, a combination of ART method and Metropolis algorithm could relax the system to a deeper $E_{\mathrm{IS}}$ level. But it will take prohibiting amounts of computing time and therefore is impractical at this moment. Given the challenge, we formulated an 'inverse' strategy in the present study. To be more specific, we first seek (via trial-and-error manner) appropriate functions of $W\left(E_{\mathrm{IS}}\right)$ and $\mu\left(E_{\mathrm{IS}}\right)$ that can produce the correct equilibrium line through equation (7), and then compare the obtained functions with the limited data points in Fig. $2 c$ to see whether they are consistent with the data or not.

The equilibrium line in supercooled liquid can be extracted from MD results during the cooling studies ${ }^{12,13}$. Figure 3 shows the variations of IS energy at different temperatures in $\mathrm{MD}$ studies at cooling rates of $10^{9} \mathrm{Ks}^{-1}$ and $10^{8} \mathrm{Ks}^{-1}$. Given the intrinsic limitation on timescale in $\mathrm{MD}$, in the present study we 


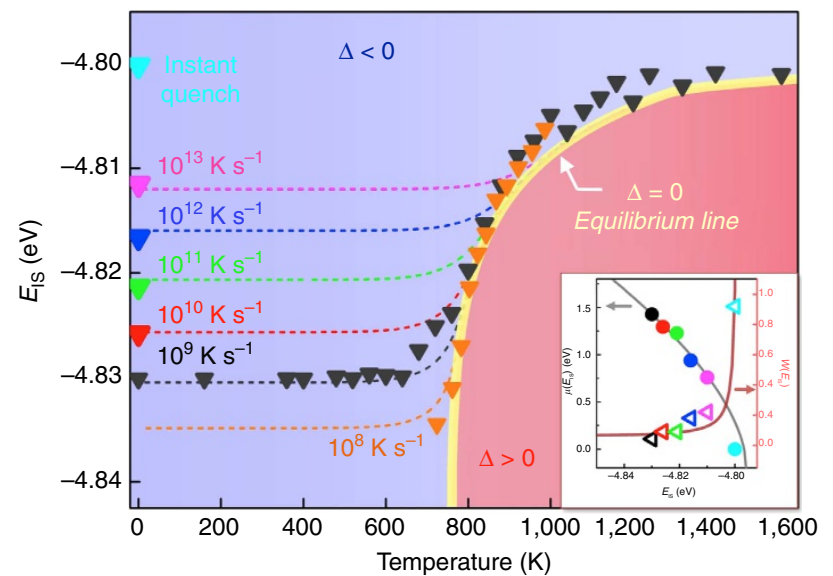

Figure 3 | Regimes of ageing and rejuvenation in IS energy-temperature space. The triangle data points are the MD results at different thermal protocols. In order to keep the plot less busy, only the final quenched IS energy results are shown for the conditions of $10^{10} \mathrm{Ks}^{-1}$ $10^{11} \mathrm{Ks}^{-1}, 10^{12} \mathrm{Ks}^{-1}, 10^{13} \mathrm{Ks}^{-1}$, and instant quench (more MD data are shown in Supplementary Fig. 3). Yellow curve is the extracted equilibrium line from MD data. To reproduce the equilibrium line through equation (7), the expected $W$ and $\mu$ should have the forms of $W\left(E_{\mathrm{IS}}\right)=0.0649 \times\left[1-\exp \left(\frac{E_{\mathrm{IS}}+4.799}{0.0153}\right)\right]^{-1}$, and $\mu\left(E_{\mathrm{IS}}\right)=12 \cdot\left(-E_{\mathrm{IS}}-4.796\right)^{0.6}-0.15$, respectively, which are shown by the two curves in the inset plot. It can be seen that the previously obtained data points in Fig. $2 \mathrm{c}$ match very well with these functions. The dashed curves are the predicted cooling curves by equation (2) at different thermal protocols. The blue area corresponds to the ageing regime since $\delta$ is negative, while the red area represents the rejuvenation regime since $\delta$ is positive.

are only able to collect small amounts of data in a narrower temperature range for the condition of $10^{8} \mathrm{Ks}^{-1}$ (orange triangles in Fig. 3). At high temperatures, the system can easily get equilibrated and the IS energy is almost constant. However below $1,000 \mathrm{~K}$, the IS energy becomes very sensitive to temperature and the required time for reaching equilibrium becomes exceedingly long as temperature decreases. When the timescale of cooling rate cannot keep up with intrinsic timescale in supercooled liquid, the system would jump out of equilibrium and eventually turn into the glassy state with a frozen IS (e.g., a flat array of black triangles at low temperatures in Fig. 3). From the MD data we can thus extract the equilibrium line, as shown by the yellow curve in Fig. 3 .

As discussed above, in order to reproduce the equilibrium line through equation (7), we found the appropriate forms and shapes of $W\left(E_{\mathrm{IS}}\right)$ and $\mu\left(E_{\mathrm{IS}}\right)$, which are shown by the two curves in the inset plot of Fig. 3 (more details in Supplementary Material Eqs 2,3 ). It can be seen that the previously obtained six sets of data points from independent ART studies are fully consistent with the assumed $W\left(E_{\mathrm{IS}}\right)$ and $\mu\left(E_{\mathrm{IS}}\right)$. Such good agreements between two independent studies validate the current model and demonstrate that the nature of ageing-rejuvenation crossover in glassy system originates from activation $\left(E_{\mathrm{A}}\right)$-relaxation $\left(E_{\mathrm{R}}\right)$ balancing in PEL. At lower temperature or higher IS energy, the average activation energy $\bar{E}_{\mathrm{A}}\left(E_{\mathrm{IS}}, T\right)$ becomes smaller, leading to a negative $\Delta$ and the ageing regime (blue area), whereas at higher temperature or lower IS energy, $\Delta$ becomes positive, corresponding to the rejuvenation regime (red area).

Origin of thermal hysteresis. To understand fully the dynamics of IS energy at arbitrary thermal protocols (including those nonequilibrium scenarios), one still needs to solve equation (2) under various conditions. As mentioned before, the entropic effect in

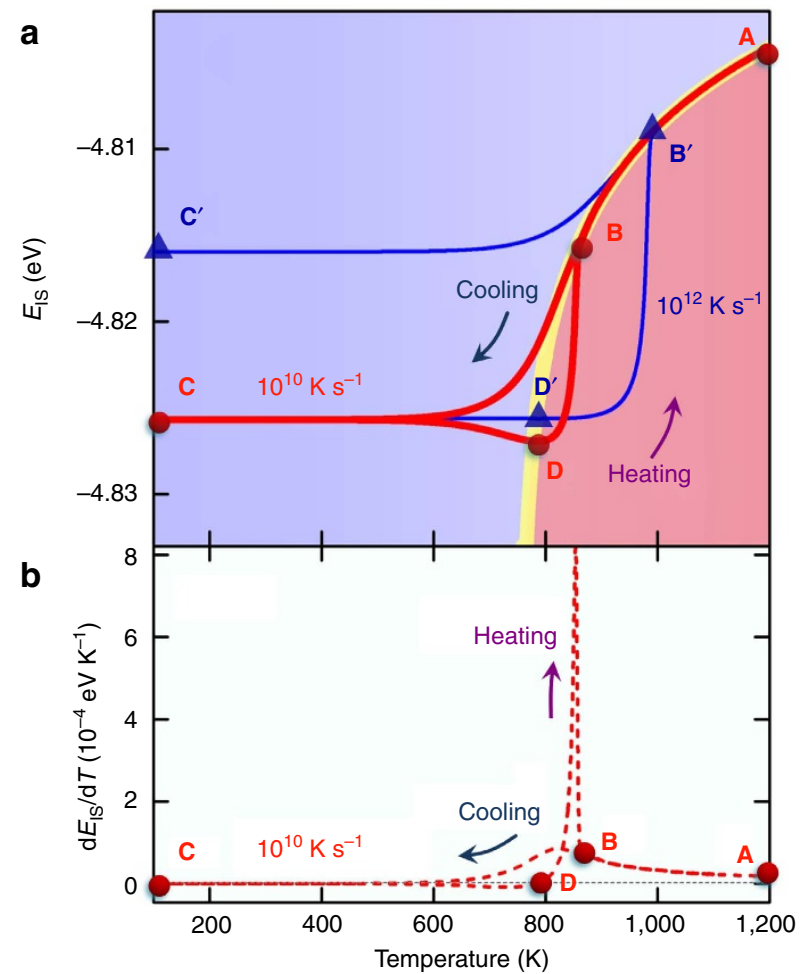

Figure 4 | Origin of thermal hysteresis in cyclic thermal scanning. (a) $E_{1 S}$ evolutions at two different thermal protocols predicted by equation (2). Red curve represents a cooling $(\mathbf{A} \rightarrow \mathbf{B} \rightarrow \mathbf{C})$ and heating $(\mathbf{C} \rightarrow \mathbf{D} \rightarrow \mathbf{B} \rightarrow \mathbf{A})$ process with the $\dot{T}$ of $10^{10} \mathrm{~K} \mathrm{~s}^{-1}$. Blue curve represents a heating $\left(\mathbf{C} \rightarrow \mathbf{D}^{\prime} \rightarrow \mathbf{B}^{\prime} \rightarrow \mathbf{A}\right)$ and cooling $\left(\mathbf{A} \rightarrow \mathbf{B}^{\prime} \rightarrow \mathbf{C}^{\prime}\right)$ process with the $\dot{T}$ of $10^{12} \mathrm{~K} \mathrm{~s}^{-1}$. (b) The derivation of $E_{\mathrm{Is}}$ with respect to $T$ associated with the first protocol shown in the upper panel. The heating process yields to a bigger peak at higher temperature than in the cooling, a known hysteresis phenomenon in glassy system.

equation (2) is included in the jump frequency as $v \propto \exp \left(S\left(E_{\mathrm{IS}}, T\right) / k_{\mathrm{B}}\right)$. As first pointed out by Goldstein ${ }^{30}$ and Johari ${ }^{31}$ and later verified in many other studies ${ }^{32-35}$, the entropy of a glassy material is dependent on both $E_{\mathrm{IS}}$ and $T$. Although the quantitative relationship varies a bit in different studies, the general rule is that entropy becomes smaller at deeper IS energy or at lower temperature (more details in Supplementary Note 3). Having considered this, the numerical solutions to IS energy at different cooling rates can then be readily calculated (dashed curves in Fig. 3), which are found fully consistent with the independent MD simulation results at the same conditions.

Thermal hysteresis near the glass transition is a well-known phenomenon in amorphous materials ${ }^{36,37}$. DSC experiments show that, with the same rate of temperature change, heating process leads to a larger heat capacity peak at a higher temperature compared to the cooling process. Here we show that such a behavior can be naturally explained by our IS energy evolution model, i.e., equation (2).

The red curve in Fig. 4 (upper panel) shows a cooling $(\mathbf{A} \rightarrow \mathbf{B} \rightarrow \mathbf{C})$ and heating $(\mathbf{C} \rightarrow \mathbf{D} \rightarrow \mathbf{B} \rightarrow \mathbf{A})$ process at the rate of $10^{10} \mathrm{~K} \mathrm{~s}^{-1}$ predicted by equation (1). In a high temperature regime (e.g., $\mathbf{A} \rightarrow \mathbf{B}$ ) the system can be equilibrated at a very short time scale and therefore the cooling curve coincides with the equilibrium line. At lower temperatures, however, the cooling rate of $10^{10} \mathrm{~K} \mathrm{~s}^{-1}$ is too fast to equilibrate the system, and the cooling curve deviates from the yellow line and enters the ageing regime (i.e., $\Delta<0$ starting from point $\mathbf{B}$. At this stage $(\mathbf{B} \rightarrow \mathbf{C}) E_{\mathrm{IS}}$ is monotonically decreasing, and the reason why it looks almost flat 
below $500 \mathrm{~K}$ is that the jump frequency $v$ becomes smaller at a deeper IS energy or at a lower temperature, resulting in a very slow change in $E_{\text {Is }}$. Now upon heating from point $\mathbf{c}, E_{\text {IS }}$ keeps monotonically decreasing until the curve hits the yellow line at point $\mathbf{D}$, because at this stage $(\mathbf{C} \rightarrow \mathbf{D})$ the system is still located in the $\Delta<0$ regime (the reason it looks flat below $500 \mathrm{~K}$ is the same as described above). This in fact is the origin of the thermal hysteresis behavior.

Beyond the point $\mathbf{D}$ the system enters the $\Delta>0$ regime and $E_{\text {IS }}$ begins to increase. Due to the entropic effect the jump frequency $v$ becomes larger as both $E_{\mathrm{IS}}$ and temperature increase, therefore the increase in the IS energy becomes faster and faster until it hits again the equilibrium line at point $\mathbf{B}$. It then follows the yellow line afterward. The dashed red curve in Fig. 4 (lower panel) is the derivative of $E_{\mathrm{IS}}$ with respect to $T$ in this cooling-heating process. Clearly the key features of thermal hysteresis, namely a larger heat capacity peak at a higher temperature during heating than in cooling, are naturally reflected in this $E_{\mathrm{IS}}$ evolution model. It is also worth noting that because the process $\mathbf{B} \rightarrow \mathbf{C} \rightarrow \mathbf{D} \rightarrow \mathbf{B}$ is an off-equilibrium process, the accurate positions of those points are sensitive to the initial conditions and thermal protocols. For example Fig. 4 shows a heating $\left(\mathbf{C} \rightarrow \mathbf{D}^{\prime} \rightarrow \mathbf{B}^{\prime} \rightarrow \mathbf{A}\right)$ and cooling $\left(\mathbf{A} \rightarrow \mathbf{B}^{\prime} \rightarrow \mathbf{C}^{\prime}\right)$ process from the same point $\mathbf{C}$, but at a different $\dot{T}$ of $10^{12} \mathrm{~K} \mathrm{~s}^{-1}$. A higher heating rate makes the minimum at point $\mathbf{D}^{\prime}$ shallower than at point $\mathbf{D}$, and pushes the fast increase in $E_{\mathrm{IS}}$ to higher temperatures (from $\mathbf{D} \rightarrow \mathbf{B}$ to $\mathbf{D}^{\prime} \rightarrow \mathbf{B}^{\prime}$ ). This also explains why higher heating rates lead to heat capacity peaks at higher temperatures $i^{36}$. Beyond the point $\mathbf{B}^{\prime}$, even with the higher $\dot{T}$ the system has enough time to reach equilibrium, and therefore $\mathbf{B}^{\prime} \rightarrow \mathbf{A}$ coincides again with the yellow line. In the cooling process afterward, the higher cooling rate $\left(10^{12} \mathrm{Ks}^{-1}\right)$ makes the curve deviate from the equilibrium line earlier and end up with a higher $E_{\mathrm{IS}}$ at point $\mathbf{C}^{\prime}$ than the initial point $\mathbf{C}$ prepared at a lower cooling rate of $10^{10} \mathrm{Ks}^{-1}$.

Anomalous peaks in truncated thermal scanning. In the regular heating-cooling protocols, glasses are heated up to a well-equilibrated liquid state far beyond the glass transition temperatures and then cooled down back to low temperatures (e.g., $\mathbf{C} \rightarrow \mathbf{D}^{\prime} \rightarrow$ $\mathbf{B}^{\prime} \rightarrow \mathbf{A} \rightarrow \mathbf{B}^{\prime} \rightarrow \mathbf{C}^{\prime}$ in Fig. 4). Under such protocols, $E_{\text {IS }}$ shows a normal and monotonic decreasing behavior during the cooling process. However if the protocol is truncated, e.g., by terminating the heating process at an intermediate point between $\mathbf{D}^{\prime}$ and $\mathbf{B}^{\prime}$ and immediately starting the cooling process afterwards, our model would predict a non-monotonic variation of $E_{\text {IS }}$ during cooling with a peak right on the activation-relaxation balancing line. The reason is again readily explained by equation (2). To be more specific let us consider a truncated protocol (at a fixed $\dot{T}$ of $10^{12} \mathrm{~K} \mathrm{~s}^{-1}$ ) starting from the same point $\mathrm{C}$ as in Fig. 4 but with a bouncing back temperature at point $\mathbf{t}$, as illustrated in the lower panel of Fig. 5.

Now when the cooling process just kicks off, because the system is still located in the $\Delta>0$ regime, $E_{\mathrm{IS}}$ keeps increasing even if the temperature is decreasing. Once the curve hits and goes across the yellow line $\Delta$ turns into negative and $E_{\mathrm{IS}}$ starts to decrease, leaving behind a peak value of $E_{\mathrm{IS}}$ right at the yellow line (point $\mathbf{p})$. Different bouncing back temperatures yield different curves, but they all display peaks at the activationrelaxation balancing line (lower panel in Fig. 5).

To verify such prediction, independent MD tests are employed at the same $\dot{T}\left(10^{12} \mathrm{~K} \mathrm{~s}^{-1}\right)$. As seen in the upper panel of Fig. 5, if the bouncing back temperature is very high (around $1,200 \mathrm{~K}$ ), the MD displays a normal protocol behavior shown by the blue triangles. However when the heating processed are truncated at

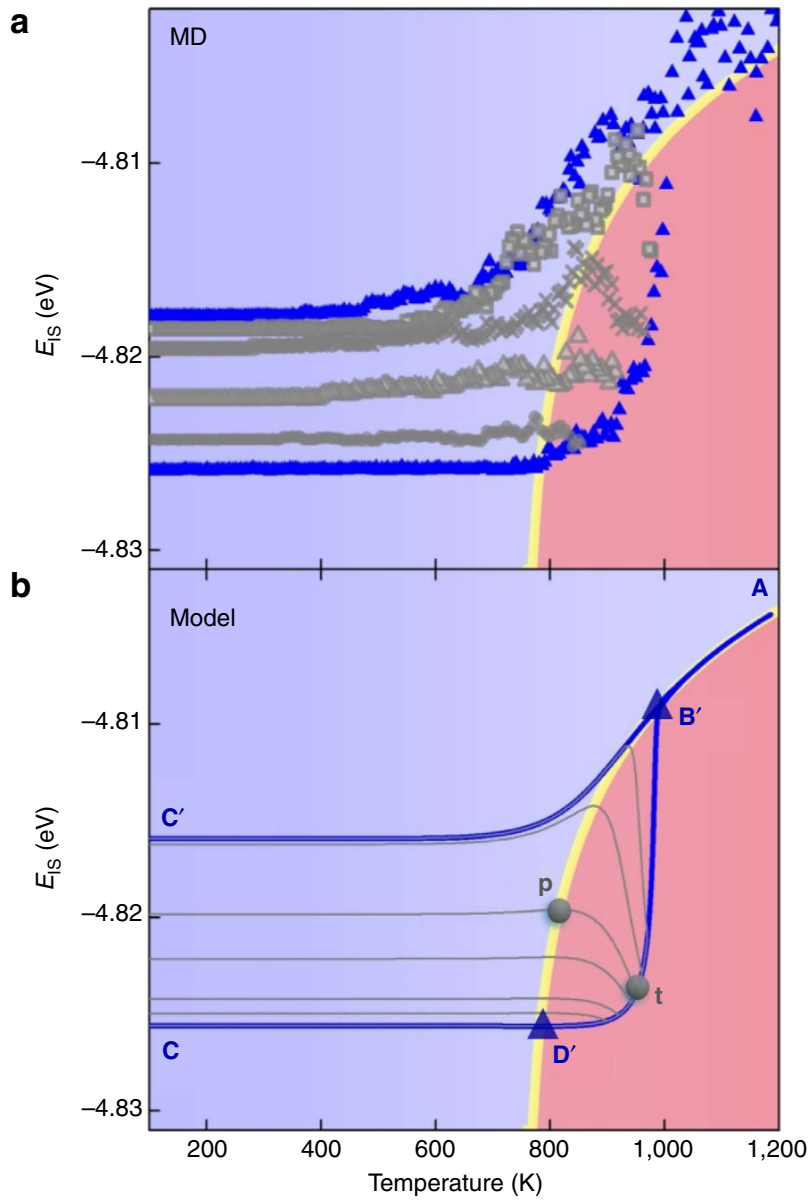

Figure 5 | Predicted abnormal peaks in truncated thermal scanning and the validations by MD results. (a) MD simulations with $\dot{T}$ of $10^{12} \mathrm{Ks}^{-1}$, and with the bouncing back temperatures at 985, 970, 920 and $860 \mathrm{~K}$, respectively (the grey data points from high to low). The MD data show clear peak structures precisely at the $E_{\mathrm{A}}-E_{\mathrm{R}}$ balancing line predicted by equation (7). (b) Model-predicted abnormal peak of $E_{\mid S}$ at point $\mathbf{p}$ on the yellow line in the truncated thermal cycle (i.e., bouncing back temperature at $\mathbf{t}$ between $\mathbf{D}^{\prime}$ and $\mathbf{B}^{\prime}$ ), which are well consistent with independent MD tests.

intermediate temperatures (between $800 \mathrm{~K}$ to $1,000 \mathrm{~K}$ ), the $\mathrm{MD}$ data (grey points) clearly show peaks precisely located at yellow line predicted by equation (6). The good agreements here again demonstrate the underlying driving force of ageing or rejuvenation comes from the interplay between activation and relaxation on the PEL.

\section{Discussion}

In the present study we develop a model to describe the dynamics of IS in glassy systems, which makes it possible to quantify the $E_{\mathrm{IS}}$ evolution in a self-consistent manner. It has been convincingly shown that system's ageing $\left(\dot{E}_{\mathrm{IS}}<0\right)$ or rejuvenation $\left(\dot{E}_{\mathrm{IS}}>0\right)$ is governed by the underlying competition between activation and relaxation on the PEL. As demonstrated in Fig. 3, the equilibrium line of the supercooled liquid is exactly the activation-relaxation balancing curve (i.e., $\Delta=0$ ). We would like to note that, in obtaining the equilibrium line one only needs to know the $E_{\mathrm{A}}$ and $E_{\mathrm{R}}$ spectra, i.e., the distributions of $P\left(E_{\mathrm{A}} \mid E_{\mathrm{IS}}\right)$ and $P\left(E_{\mathrm{R}} \mid E_{\mathrm{IS}}\right)$. Because such distributions are simultaneously determined by the PEL and interactions between constituent particles in the materials, from this sense the model shown in the present work 
does not need to introduce pre-assumed mechanisms or further parameters, which could be an advantage for disordered materials modeling.

As shown in equation (5) and Supplementary Fig. 1, the distribution of the relaxation energy after passing through the saddle point is almost always the same, regardless of the initial state. The same feature was also observed in amorphous $\mathrm{Si}$ (ref. 17), and was suggested by internal friction experiments in a metallic glass as well ${ }^{38}$. In other words, by the time the system reaches the saddle point it forgets where it came from. Therefore, the correlations between elementary activations and relaxations in PEL, if any, are very weak, as evidenced by Supplementary Fig. 2 and in earlier studies by Swayamjyoti et al. ${ }^{39,40}$ and Kallel et al. ${ }^{17}$. The decoupling of activation and relaxation greatly simplifies the dynamics of IS migration through equation (2). Further implications of this critical feature will be discussed elsewhere. We would like to note that, the quantitative results in the present work rely upon two assumptions: (i) the independence of $\bar{E}_{\mathrm{R}}$ on $\bar{E}_{\mathrm{A}}$ and $E_{\mathrm{IS}}$, and (ii) the accuracy of $P\left(E_{\mathrm{A}} \mid E_{\mathrm{IS}}\right)$ over the entire range of $E_{\mathrm{IS}}$. Although the comparison with $\mathrm{MD}$ results in the cooling rate ranging from $10^{13} \mathrm{~s}^{-1}$ to $10^{8} \mathrm{~s}^{-1}$ suggest the two assumptions are valid, there is no guarantee that they still hold at lower levels of $E_{\mathrm{IS}}$ (i.e., at much lower cooling rates). However, we believe that the underlying framework of the present model, i.e., equation (2), is still valid, because equation (2) only describes the physics process of hopping in PEL and does not rely on the above mentioned two assumptions. Even if at deep levels of $E_{\mathrm{IS}}$ (e.g., at experimental quench rates) $P\left(E_{\mathrm{A}} \mid E_{\mathrm{IS}}\right)$ were to be significantly different, and $\bar{E}_{\mathrm{R}}$ were to depend on $E_{\mathrm{IS}}$ and correlate with $\bar{E}_{\mathrm{A}}$, equation (2) would still provide a steady-state solution, although the detailed shape and position of such equilibrium line in $E_{\mathrm{IS}}-T$ diagram could be different from what is shown now in Fig. 3 and given by equation (7).

The $E_{\mathrm{IS}}$ evolution model in the present study can also naturally explain the thermal hysteresis phenomenon observed in experiments. In addition, our model predicts an anomalous peak of $E_{\mathrm{IS}}$ in truncated thermal cycles, which has been verified by independent MD studies shown in Fig. 5. Such a success in prediction suggests that our model correctly captures the interplay between activation and relaxation on the PEL of the system, which are the underlying driving force in supercooled liquids and glasses. This in turn allows us to speculate about possible implications of such findings. For example during the stage $\mathbf{t} \rightarrow \mathbf{p}$ in Fig. 5, the IS energy keeps increasing even if the temperature is being reduced. This corresponds to negative heat capacity which might be captured in high-throughput DSC measurement. Interestingly experiments have found some nonmonotonic behaviors in glassy systems under different thermal protocols, such as the crossover phenomena ${ }^{41,42}$ and the Kovac effect $^{43}$. The most straightforward explanation for the crossover is that there should be more than one kinetic processes involved $^{41,42}$. In other words a broad distribution of $E_{\mathrm{A}}$ is critical, and our present results provide fundamental support to such hypothesis.

It is also worth noting that in the present study we consider a metallic glass system $\mathrm{Cu}_{56} \mathrm{Zr}_{44}$ known as a fragile glass former, and its $E_{\mathrm{A}}$ spectra show two-mode decomposition. Due to the persistence of $\mathrm{M} 1$ mode, there exists a critical temperature $\left(T_{\mathrm{c}} \sim 760 \mathrm{~K}\right)$ below which there is no solution to equation (7). In other words, below $T_{c}$ equilibrium can never be reached, or equivalently ergodicity is broken. However recent studies ${ }^{17}$ on a strong glass former, amorphous silicon, showed that $E_{\mathrm{A}}$ spectra display only the M2 mode. Very interestingly, we find that if M1 is missing then there always is a solution to equation (7), or equivalently $T_{\mathrm{c}} \sim 0 \mathrm{~K}$. The asymptotic behavior as the system approaching $T_{\mathrm{c}}$ can also be explicitly obtained based on details of
$P\left(E_{\mathrm{A}} \mid E_{\mathrm{IS}}\right)$ and $P\left(E_{\mathrm{R}} \mid E_{\mathrm{IS}}\right)$, as will be discussed later in a separate paper. It is also worth noting that, the well-known VogelTammann-Fulcher (VFT) relationship $\left(\eta=A \exp \left[B /\left(T-T_{0}\right)\right]\right)$ correlates the fragility of glass with a parameter $T_{0}$, where $T_{0}$ is a finite number for a fragile system and $T_{0}$ approaches to $0 \mathrm{~K}$ for a strong system. The similarity between $T_{0}$ and $T_{c}$ thus builds very interesting and profound connections between the $E_{\mathrm{A}}$ spectra and the origin of fragility in glass formers. Admittedly there are new challenges remain to be addressed. For example, in the VFT model the $T_{0}$ is attributed to the properties of the mega-basins in $\mathrm{PEL}^{13}$. But in the present work, with only neighboring basins considered, a similar role of $T_{\mathrm{c}}$ can also be obtained. Reconciling the two pictures, as well as identifying the structural correspondence to the different modes of M1 and M2, would warrant further studies.

\section{Methods}

Model set-up and art parameters. The studied $\mathrm{Cu}_{56} \mathrm{Zr}_{44}$ system contains 2,000 atoms, with an embedded-atom method (EAM) interaction ${ }^{18}$. The sizes of the system are $32.43 \AA \times 32.43 \AA \times 32.43 \AA$, with periodic boundary conditions employed to all three directions. In ART simulations, the initial perturbations are introduced to a small group of atoms with local connectivity ${ }^{44}$. Specifically, an atom is selected as the central atom, then random displacements are induced to this atom and its first nearest neighbors. The perturbation direction is randomly chosen, while the magnitude of displacement is fixed at $0.5 \AA$. The system is relaxed to the saddle point following the Lanczos algorithm ${ }^{15,45-47}$, when the curvature of PEL is less than $-0.01 \mathrm{eV} \AA^{-2}$. The convergence to saddle point is reached when the force of the system is less than $0.05 \mathrm{eV}^{-1}$. For each selected group of atoms, 10 ART searches with different random perturbation directions are employed. The 2,000 atoms system thus provides 20,000 searches in ART. Therefore six samples together contribute 120,000 ART searches. After removing the failed and redundant searches, on average around 2,500 different processes are identified for each sample.

Data availability. The data that support the findings of this study are available from the corresponding author upon reasonable request.

\section{References}

1. Wisitsorasak, A. \& Wolynes, P. G. On the strength of glasses. Proc. Natl Acad. Sci. 109, 16068-16072 (2012).

2. Cheng, Y. Q. \& Ma, E. Atomic-level structure and structure-property relationship in metallic glasses. Prog. Mater. Sci. 56, 379-473 (2011).

3. Chen, M. Mechanical behavior of metallic glasses: microscopic understanding of strength and ductility. Annu. Rev. Mater. Res. 38, 445-469 (2008).

4. Wakeda, M., Saida, J., Li, J. \& Ogata, S. Controlled rejuvenation of amorphous metals with thermal processing. Sci. Rep. 5, 10545 (2015).

5. Rehwald, C. et al. Aging effects manifested in the potential-energy landscape of a model glass former. Phys. Rev. E 82, 021503 (2010)

6. Berthier, L. \& Biroli, G. Theoretical perspective on the glass transition and amorphous materials. Rev. Mod. Phys. 83, 587-645 (2011).

7. Cohen, M. H. \& Turnbull, D. Molecular transport in liquids and glasses. J. Chem. Phys. 31, 1164-1169 (1959)

8. Spaepen, F. A microscopic mechanism for steady state inhomogeneous flow in metallic glasses. Acta Metall. 25, 407-415 (1977).

9. Falk, M. L. \& Langer, J. S. Dynamics of viscoplastic deformation in amorphous solids. Phys. Rev. E 57, 7192-7205 (1998).

10. Lacks, D. J. \& Osborne, M. J. Energy landscape picture of overaging and rejuvenation in a sheared glass. Phys. Rev. Lett. 93, 255501 (2004).

11. Stillinger, F. H. A topographic view of supercooled liquids and glass formation. Science 267, 1935-1939 (1995).

12. Sastry, S., Debenedetti, P. G. \& Stillinger, F. H. Signatures of distinct dynamical regimes in the energy landscape of a glass-forming liquid. Nature 393, 554-557 (1998).

13. Debenedetti, P. G. \& Stillinger, F. H. Supercooled liquids and the glass transition. Nature 410, 259-267 (2001).

14. Barkema, G. T. \& Mousseau, N. Event-based relaxation of continuous disordered systems. Phys. Rev. Lett. 77, 4358-4361 (1996).

15. Cances, E., Legoll, F., Marinica, M. C., Minoukadeh, K. \& Willaime, F. Some improvements of the activation-relaxation technique method for finding transition pathways on potential energy surfaces. J. Chem. Phys. 130, 114711 (2009).

16. Rodney, D. \& Schuh, C. Distribution of thermally activated plastic events in a flowing glass. Phys. Rev. Lett. 102, 235503 (2009).

17. Kallel, H., Mousseau, N. \& Schiettekatte, F. Evolution of the potential-energy surface of amorphous silicon. Phys. Rev. Lett. 105, 045503 (2010). 
18. Cheng, Y. Q., Ma, E. \& Sheng, H. W. Atomic level structure in multicomponent bulk metallic glass. Phys. Rev. Lett. 102, 245501 (2009).

19. Rodney, D. \& Schrøder, T. On the potential energy landscape of supercooled liquids and glasses. Eur. Phys. J. E 34, 1-7 (2011).

20. Rodney, D., Tanguy, A. \& Vandembroucq, D. Modeling the mechanics of amorphous solids at different length scale and time scale. Model. Simul. Mater. Sci. Eng. 19, 083001 (2011).

21. Fan, Y., Iwashita, T. \& Egami, T. How thermally activated deformation starts in metallic glass. Nat. Commun. 5, 5083 (2014).

22. Fan, Y., Iwashita, T. \& Egami, T. Evolution of elastic heterogeneity during aging in metallic glasses. Phys. Rev. E 89, 062313 (2014)

23. Fan, Y., Iwashita, T. \& Egami, T. Crossover from localized to cascade relaxations in metallic glasses. Phys. Rev. Lett. 115, 045501 (2015).

24. Delogu, F. Thermal and mechanical activation of inelastic events in metallic glasses. Scr. Mater. 113, 145-149 (2016).

25. Tsamados, M. Plasticity and dynamical heterogeneity in driven glassy materials. Eur. Phys. J. E 32, 165-181 (2010)

26. Argon, A. S. \& Kuo, H. Y. Free energy spectra for inelastic deformation of five metallic glass alloys. J. Non-Crystal. Solids 37, 241-266 (1980).

27. Derlet, P. M. \& Maaß, R. Linking high- and low-temperature plasticity in bulk metallic glasses: thermal activation, extreme value statistics and kinetic freezing. Philos. Mag. 93, 4232-4263 (2013).

28. Derlet, P. M. \& Maaß, R. Linking high- and low-temperature plasticity in bulk metallic glasses II: use of a log-normal barrier energy distribution and a mean-field description of high-temperature plasticity. Philos. Mag. 94, 2776-2803 (2014)

29. Shimizu, F., Ogata, S. \& Li, J. Yield point of metallic glass. Acta. Mater. 54, 4293-4298 (2006).

30. Goldstein, M. Viscous liquids and the glass transition. V. Sources of the excess specific heat of the liquid. J. Chem. Phys. 64, 4767-4774 (1976).

31. Johari, G. P. On the heat capacity, entropy and 'glass transition' of vitreous ice. Philos. Mag. 35, 1077-1090 (1977).

32. Yan, Q., Jain, T. S. \& de Pablo, J. J. Density-of-states Monte Carlo simulation of a binary glass. Phys. Rev. Lett. 92, 235701 (2004).

33. Sastry, S. The relationship between fragility, configurational entropy and the potential energy landscape of glass-forming liquids. Nature 409, 164-167 (2001).

34. Johari, G. P. A resolution for the enigma of a liquid's configurational entropymolecular kinetics relation. J. Chem. Phys. 112, 8958-8969 (2000).

35. Koziatek, P., Barrat, J.-L., Derlet, P. \& Rodney, D. Inverse Meyer-Neldel behavior for activated processes in model glasses. Phys. Rev. B 87, 224105 (2013).

36. Dyre, J. C. Colloquium: the glass transition and elastic models of glass-forming liquids. Rev. Mod. Phys. 78, 953-972 (2006)

37. Wei, S., Gallino, I., Busch, R. \& Angell, C. A. Glass transition with decreasing correlation length during cooling of Fe50Co50 superlattice and strong liquids. Nat. Phys. 7, 178-182 (2011).

38. Morito, N. \& Egami, T. Internal friction and reversible structural relaxation in the metallic glass Fe32Ni36Cr14P12B6. Acta Metall. 32, 603-613 (1984).

39. Swayamjyoti, S., Löffler, J. F. \& Derlet, P. M. Local structural excitations in model glasses. Phys. Rev. B 89, 224201 (2014).

40. Swayamjyoti, S., Löffler, J. F. \& Derlet, P. M. Local structural excitations in model glass systems under applied load. Phys. Rev. B 93, 144202 (2016).

41. Greer, A. L. \& Leake, J. A. Structural relaxation and crossover effect in a metallic glass. J. Non-Crystal. Solids 33, 291-297 (1979).
42. Boesch, L., Napolitano, A. \& Macedo, P. B. Spectrum of volume relaxation times in $\mathrm{B}_{2} \mathrm{O}_{3}$. J. Am. Ceram. Soc. 53, 148-153 (1970).

43. Kovacs, A. J., Aklonis, J. J., Hutchinson, J. M. \& Ramos, A. R. Isobaric volume and enthalpy recovery of glasses. II. A transparent multiparameter theory. J. Polym. Sci. 17, 1097-1162 (1979).

44. Iwashita, T., Nicholson, D. M. \& Egami, T. Elementary excitations and crossover phenomenon in liquids. Phys. Rev. Lett. 110, 205504 (2013).

45. Béland, L. K., Brommer, P., El-Mellouhi, F., Joly, J.-F. \& Mousseau, N. Kinetic activation-relaxation technique. Phys. Rev. E 84, 046704 (2011).

46. Mousseau, N. et al. The activation-relaxation technique: ART nouveau and kinetic ART. J. At. Mol. Opt. Phys. 2012, 14 (2012).

47. Jean-François, J., Laurent Karim, B., Peter, B., Fedwa, E.-M. \& Normand, M. Optimization of the kinetic activation-relaxation technique, an off-lattice and self-learning kinetic Monte-Carlo method. J. Phys. 341, 012007 (2012).

\section{Acknowledgements}

The authors are grateful to M. Atzmon and J.S. Langer for useful discussion. This work was supported by the U.S. Department of Energy, Office of Science, Basic Energy Sciences, Materials Science and Engineering Division. Y.F. would also like to thank the support by start-up funding from University of Michigan, Ann Arbor.

\section{Author contributions}

Y.F. conceived the model. Y.F. and T.E. designed the study. Y.F. performed the ART simulations and analyzed data. T.I. performed MD studies at different cooling rates. Y.F., T.I. and T.E. all contributed to discussions and wrote the paper.

\section{Additional information}

Supplementary Information accompanies this paper at http://www.nature.com/ naturecommunications

Competing interests: The authors declare no competing financial interests.

Reprints and permission information is available online at http://npg.nature.com/ reprintsandpermissions/

How to cite this article: Fan, Y. et al. Energy landscape-driven non-equilibrium evolution of inherent structure in disordered material. Nat. Commun. 8, 15417 doi: 10.1038/ncomms15417 (2017)

Publisher's note: Springer Nature remains neutral with regard to jurisdictional claims in published maps and institutional affiliations.

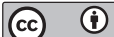

This work is licensed under a Creative Commons Attribution 4.0 International License. The images or other third party material in this article are included in the article's Creative Commons license, unless indicated otherwise in the credit line; if the material is not included under the Creative Commons license, users will need to obtain permission from the license holder to reproduce the material To view a copy of this license, visit http://creativecommons.org/licenses/by/4.0/

C) The Author(s) 2017 